\title{
ANDROGRAPHOLIDE AND ITS DERIVATIVE - A STORY OF ANTIMALARIAL DRUG DESIGN AND SYNTHESIS
}

\author{
ANDRIANOPSYAH MAS JAYA PUTRA ${ }^{1,2}$, CHAIDIR $^{3}$, MUHAMMAD HANAFI ${ }^{2}$, YUANJIANG PAN $^{4}$, ARRY YANUAR $^{1 *}$ \\ ${ }^{1}$ Department of Pharmacy, Faculty of Pharmacy, Universitas Indonesia, Depok, Indonesia. ${ }^{2}$ Division of Natural Products-Food- \\ Pharmaceuticals, LIPI Research Center for Chemistry, Tangerang Selatan, Indonesia. ${ }^{3}$ BPPT Center for Pharmaceutical and Medical \\ Technology, Tangerang Selatan, Indonesia. ${ }^{4}$ Department of Chemistry, Laboratory of Natural and Biochemistry, Zhejiang University, \\ Hangzhou, P.R. China. Email: arry.yanuar@ui.ac.id
}

Received: 21 April 2017, Revised and Accepted: 18 August 2017

\section{ABSTRACT}

Objective: Andrographolide was found to show moderate antimalarial activity against chloroquine-resistant strain of Plasmodium falciparum (PF). It thus becomes an interesting lead for new antimalarial drugs. This study describes a molecular docking of andrographolide and its derivative into the best PF geranylgeranyl pyrophosphate synthase (PFGGPPS) model.

Methods: A comparative modeling of PFGGPPS based on a crystal structure of Plasmodium vivax GGPPS was optimized and conducted. This model was considered suitable for molecular docking. Partition coefficient of andrographolide was determined to assist its derivative design based on hydrophobicity property. Synthesis of the antimalarial drug was scaled up to $5 \mathrm{~mm}$ and identified by ${ }^{13} \mathrm{C}$ - and ${ }^{1} \mathrm{H}$-nuclear magnetic resonance (NMR) spectroscopy.

Results: The optimal comparative modeling of PFGGPPS was conducted on chain B (3PH7 chain B). The calculated coefficient partition of andrographolide's derivative was higher $(+1.89)$, compared to that of andrographolide of +1.62 . The NMR data of second and third synthesis experiments were consistent at the 5 -mmol scale.

Conclusions: On the molecular docking of andrographolide into the model, an antimalarial andrographolide derivative design that is more hydrophobic than andrographolide was proposed since the stronger hydrophobicity property of drug, the better of its activity of the drug. Synthesis of this derivative with a simple and green procedure was found to be reproducible.

Keywords: Plasmodium falciparum, Geranylgeranyl pyrophosphate synthase, Andrographolide, Comparative modeling, Molecular docking, Drug design and synthesis.

(C) 2017 The Authors. Published by Innovare Academic Sciences Pvt Ltd. This is an open accessarticle under the CC BY license (http://creativecommons. org/licenses/by/4. 0/) DOI: http://dx.doi.org/10.22159/ijap.2017.v9s1.55_61

\section{INTRODUCTION}

Plasmodium falciparum (PF), a microorganism that belongs to the phylum Apicomplexa [1], is the most common cause of malaria in Indonesia [2]. The first-line treatment for uncomplicated PF malaria in the country is the artesunate-amodiaquine combination [2]. However, a failure rate of more than $10 \%$, associated with drug resistance, was reported for this treatment in Indonesia for the period 2000-2007 [2]. This report presents the basis for a new antimalarial drug development in Indonesia. Geranylgeranyl pyrophosphate synthase (GGPPS) is a key enzyme in PF isoprenoid biosynthesis [3]. It catalyzes the synthesis of $\mathrm{GGPP}^{3}$ as well as farnesyl pyrophosphate [3]. In the presence of $\mathrm{Mg}^{2+}$ cofactors [4]. It is recommended as an antimalarial drug target due to its amenability to high-throughput screening, druggability, and essentiality [5].

Andrographolide (Fig. 1) is the major secondary metabolite in an Indonesian herb, Sambiloto (Andrographis paniculata) [6]. It was found to have a moderate antimalarial activity against a chloroquine-resistant strain of $\mathrm{PF}$ [7], making it an interesting lead for new antimalarial drugs. Since it is biosynthesized from and similar to GGPP [8], it is reasonable to hypothesize that andrographolide exerts its antimalarial activity by inhibition of GGPPS. To establish this hypothesis by molecular docking, a crystal structure of PFGGPPS is needed, but this is not yet available.

Meanwhile, high-resolution GGPP-binding crystal structures of Plasmodium vivax GGPPS (PVGGPPS) are available (Protein Data Bank [PDB] 3CC9 [9] and 3PH7 [4]), based on a sequence available at UniProt [10] (A5K4U6 [11]). A basic local alignment search tool search on UniProt for this sequence gave a PFGGPPS sequence (Q86GK8 [12]) that was evidenced from transcript level. A $>70 \%$ similarity between the two sequences was revealed in our preliminary sequence alignment by ClustalX 2.1 [13]. Therefore, it is feasible to make a comparative modeling of PFGGPPS using one the PVGGPPS crystal structures as the template.

In this paper, we report the aforementioned comparative modeling. Then, we describe a molecular docking of andrographolide into the best PFGGPPS model. Based on the results of this molecular docking, we propose an antimalarial andrographolide derivative design (Fig. 1), and we report our attempt to synthesize it.

\section{METHODS}

\section{Comparative modeling of PFGGPPS}

The comparative modeling was conducted by Modeller $9 \mathrm{v}^{16}$ interfaced by EasyModeller 4.0 [16]. A sequence of PFGGPPS downloaded from UniProt [10] as a FASTA file (Q86GK8 [12]) was employed as the query. A $2.5 \AA$-resolution GGPP-binding crystal structure of PVGGPPS downloaded from the Research Collaboratory for Structural Bioinformatics (RCSB). PDB [17] as a PDB file (PDB 3PH7) [14] was selected as the template. During the comparative modeling, heteroatoms were not included, and loops were not refined. Instead, the model with the lowest discrete optimized protein energy (DOPE) score was optimized by default parameters.

\section{Preparation of PFGGPPS for molecular docking}

The optimized model of PFGGPPS from the comparative modeling was prepared for molecular docking by OEDocking 3.0.1 [18]. Detection of its active site was carried out using a molecular method, which 
was guided by Imprint Pocket [19]. The largest cavity indicated by Imprint Pocket was selected as the active site. Detection sensitivity was adjusted manually to ensure that all active site residues were covered. The molecular docking grid box was defined as $26.33 \times 43.00 \times 30.33$ $\AA^{3}=34,347 \AA^{3}$. Site shape potential was balanced (extended toward solvent and protein), and outer contour was set to $1,747 \AA^{3}$. No constraint was applied.

Preparation of andrographolide and its derivative for molecular docking

A crystal structure of andrographolide (1) in CIF format [20] was used as a starting conformation of andrographolide. The format was converted into PDB by PyMOL 1.3 [21], and then this was converted into OpenEye native format (oeb) by VIDA 4.2.1 [22]. The structure of the andrographolide's derivative (2) was built on this crystal structure by VIDA 4.2.1 [22]. Before molecular docking, every ligand was fixed to $\mathrm{pKa}=7.0$ and their tautomers assigned by QUACPAC 1.6.3.1 [23,24]. Then, the derivative was filtered for drug-likenesses and its conformations generated by OMEGA 2.5.1.4 [25] using default parameters.

\section{Molecular docking of andrographolide and its derivative}

Molecular docking of the prepared ligands was conducted by OEDocking 3.0.1 [18] in vacuo using default parameters. Every best docking pose was then subjected to optimization by SZYBKI 1.8.0.2 [26], considering a minimum distance of $2 \AA$ between PFGGPPS active site residues and the ligand. In this optimization, protein-ligand interaction energies were calculated using Merck molecular force field (MMFF) van der Waals potential, in which MMFF Coulomb was digitized on the grid.

Calculation of partition coefficient of andrographolide and its derivative

A calculation of partition coefficient (log P) of andrographolide was conducted by ACD/Labs 12.01 [15] to assist the design of 2 .

\section{Synthesis of andrographolide derivative}

Andrographolide ( $\geq 98 \%$ ) was purchased from Xi'an App ChemBio(Tech) Co., Ltd., P.R. China, and would expire on May 24, 2015. It appears as a white powder that dissolves in chloroform, ethyl acetate, and methanol $[27,28]$, and it decomposes at $70^{\circ} \mathrm{C}[29]$.

To modify 1 into 2 , andrographolide $(1 \mathrm{mmol})$ was dissolved in methanol and reacted with anhydrous sodium carbonate $(1 \mathrm{mmol})$ at $50^{\circ} \mathrm{C}$ for $2 \mathrm{hrs}$, a procedure adapted from Pandeti et al. [30] The reaction was monitored by thin-layer chromatography every hour. The desired product was purified by solvent-solvent extraction (chloroformwater 1:1), which was followed by column chromatography (eluent: $100 \%$ ethyl acetate) and identified by ${ }^{13} \mathrm{C}$ - and ${ }^{1} \mathrm{H}$-nuclear magnetic resonance (NMR) spectroscopy. The same experiment was repeated to assess reproducibility. Following a good confirmation, the procedure

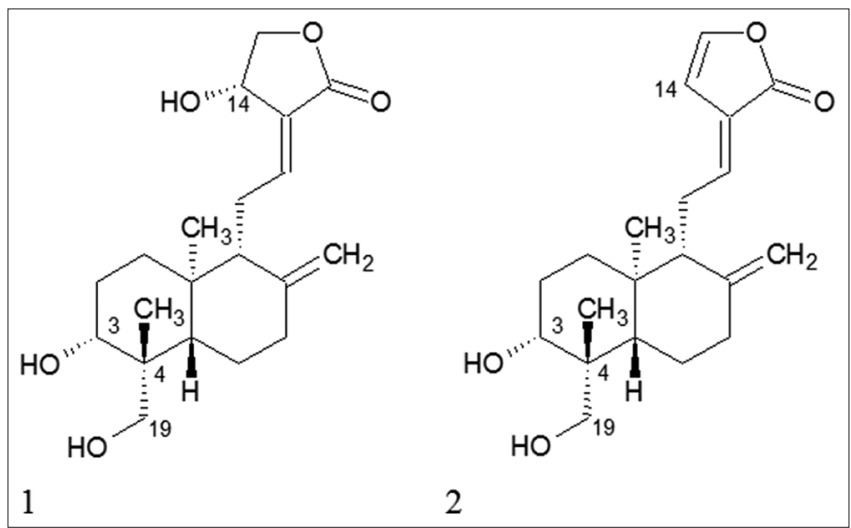

Fig. 1: Andrographolide (1) [14] and its proposed derivative (2) (drawn by ACD/Labs 12.01) [15] was scaled up to $5 \mathrm{mmol}$. Only at this point was the reaction yield calculated.

\section{RESULTS AND DISCUSSION}

\section{Comparative modeling of PFGGPPS}

Before the comparative EasyModeller, Modeler, aligned sequences of the template and query. For chains A to D of the template, the alignment scores were 265257.6250, 273733.8750, 252205.1562, and 260121.5312, respectively. Since chain B of the template gained the highest score for the alignment, and no gap presented at predicted secondary structures of the query (Fig. 2), the comparative modeling was conducted on chain B.

The comparative modeling produced seven models with the following DOPE scores: $\quad-45876.36719,-45724.93750,-45170.59375$, $-45537.06250,-45908.73047,-45727.52344$, and -45883.46484 . Model 5 received the lowest DOPE score, so it was optimized. After optimization of this model, a Ramachandran plot revealed only a few residues outside the allowed regions (Fig. 3). One of these residues was part of the PFGGPPS active site, according to Imprint Pocket (Fig. 2). Therefore, this PFGGPPS model was considered suitable for molecular docking. A comparison between the PVGGPPS crystal structure and PFGGPPS model is depicted in Fig. 4.

Molecular docking of andrographolide and its derivative The active site of PVGGPPS consists of a hydrophobic anchor $\left(\mathrm{L}^{118}, \mathrm{Q}^{119}\right.$, $\mathrm{A}^{120}, \mathrm{~F}^{122}, \mathrm{~V}^{156}, \mathrm{Y}^{160}, \mathrm{~T}^{188}$, and $\mathrm{I}^{192}$; numbering differs 20 amino acids more than sequence numbering) and a catalytic pocket containing the first aspartate-rich motif (FARM; ${ }^{126}$ DDIMD ${ }^{130}$ ) and second aspartate-rich motif (SARM; ${ }^{287}$ DDYID $^{291}$ ) [4]. GGPP positions itself in the PVGGPPS

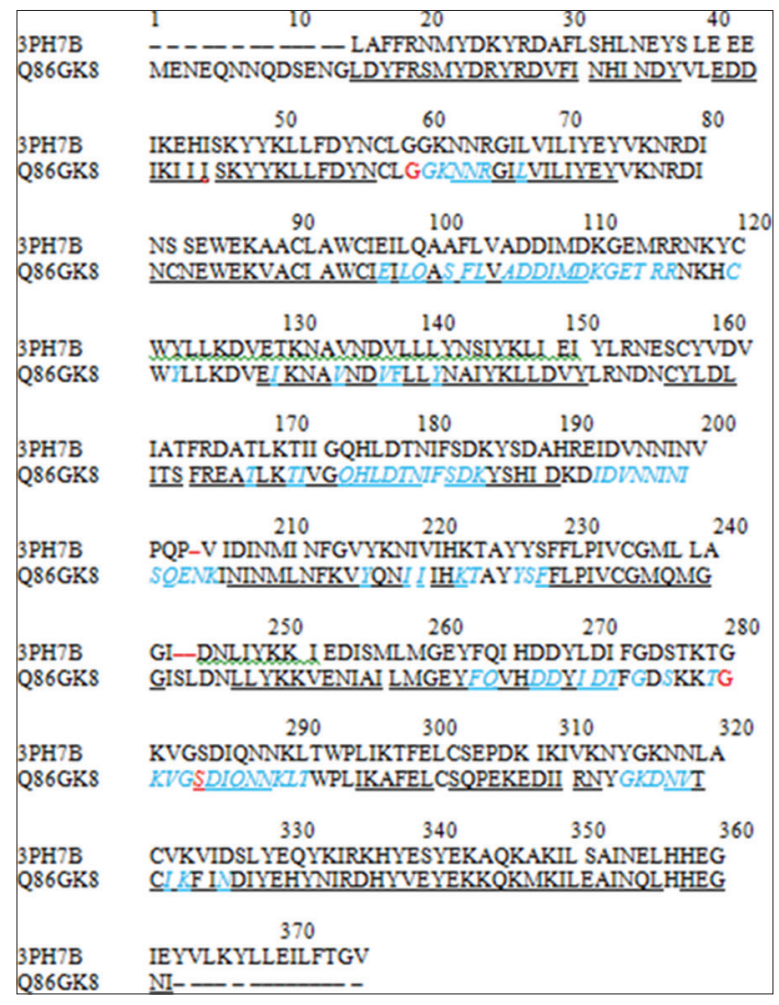

Fig. 2: Sequence alignment of the template (3PH7 chain $\mathrm{B}$ ) and query (Q86GK8). Gaps in the alignment are indicated by red dash; predicted alpha helix structures for the query are constructed by underlined amino acids; no beta structure was predicted for the query by modeller; the active Plasmodium falciparum geranylgeranyl pyrophosphate synthase site, according to Imprint

Pocket, is constructed by italicized amino acids; outliers in the Ramachandran plot are colored red in Fig. 3 
active site such that its hydrophobic part is held by the anchor while its pyrophosphate group interacts with the catalytic pocket [4]. In our docking pose of andrographolide, the hydroxyl groups at C-3 and C-19 pointed toward the SARM of PFGGPPS, while its lactone group pointed toward the hydrophobic anchor (Fig. 5).

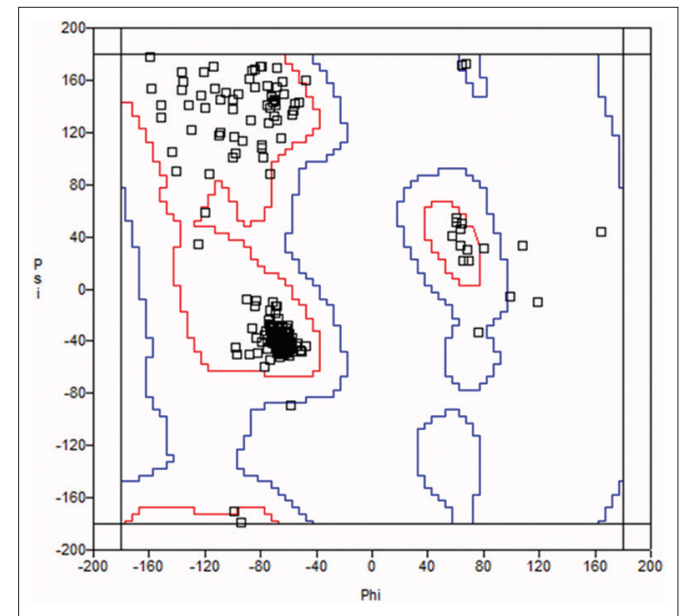

Fig. 3: Ramachandran plot of the optimized Plasmodium falciparum geranylgeranyl pyrophosphate synthase model 5

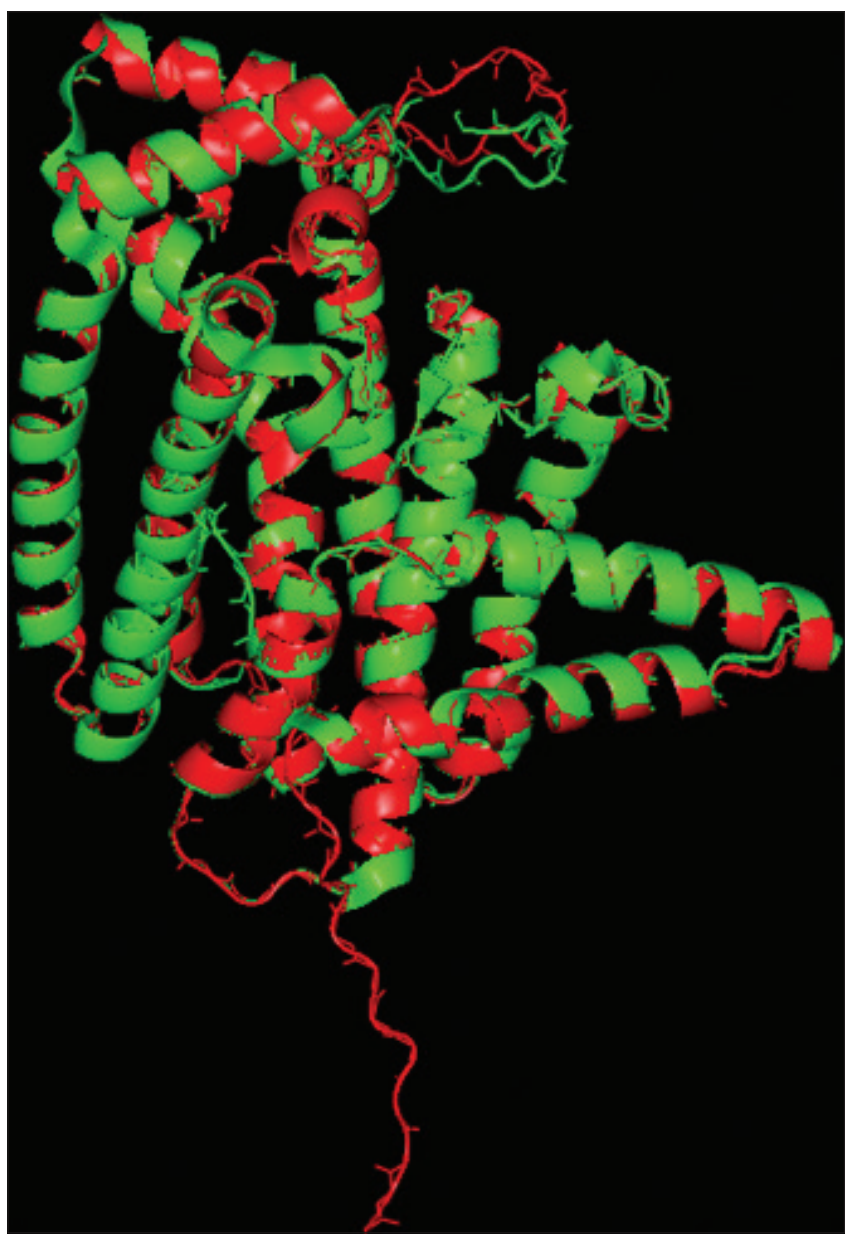

Fig. 4: Comparison between the Plasmodium vivax geranylgeranyl pyrophosphate synthase crystal structure (green) and our Plasmodium falciparum geranylgeranyl pyrophosphate synthase model (red) (image generated by PyMOL 1.3) [21]
The overall energy of the interaction between andrographolide and PFGGPPS was $-70.81114 \mathrm{kcal} / \mathrm{mol}$ (comprising electrostatic and van der Waals interactions of -58.43179 and $-12.37936 \mathrm{kcal} / \mathrm{mol}$, respectively). Stronger electrostatic interactions should be expected in the presence of $\mathrm{Mg}^{2+}$ cofactors and water molecules. This finding suggests that the andrographolide hydroxyl groups at C-3 and C-19 were essential for hydrophilic interactions, while its lactone group could be modified to become more hydrophobic.

To examine this suggestion, we proposed a design for an andrographolide derivative (2) which was more hydrophobic than andrographolide at its lactone group. On docking into PFGGPPS, this derivative gave a highly similar position and orientation (Fig. 5). Its affinity toward PFGGPPS was lost slightly, as indicated by a decrease in the overall ligand-protein interaction energy (down by about an $8^{\text {th }}$, to $-61.90828 \mathrm{kcal} / \mathrm{mol}$ ). However, the van der Waals contribution in the interaction energy increased by approximately one-third, to $-16.40830 \mathrm{kcal} / \mathrm{mol}$, suggesting that the pre-modified lactone group at two bound more tightly to the hydrophobic anchor of PFGGPPS.

Since an antimalarial drug should penetrate the membrane of PF cell and work within the cell, its activity is part determined by its hydrophobicity, which is reflected by its partition coefficient ( $\log P$ ): The higher its $\log$ P (until +5.00 , according to Lipinski's Rule of Five) [31], the better it penetrates the membrane and the higher is its activity. The calculated $\log \mathrm{P}$ of 2 is +1.89 , compared to that of andrographolide of +1.62 . Therefore, 2 is expected to penetrate the membrane better. This pharmacokinetic profile could compensate the previous pharmacodynamic one.

\section{Synthesis of the andrographolide derivative}

The above procedure was selected because it is considered simple and sufficiently green, so it is suitable for drug synthesis. Below is the result of the ${ }^{13} \mathrm{C}$ - and ${ }^{1} \mathrm{H}-\mathrm{NMR}$ for the first experiment:

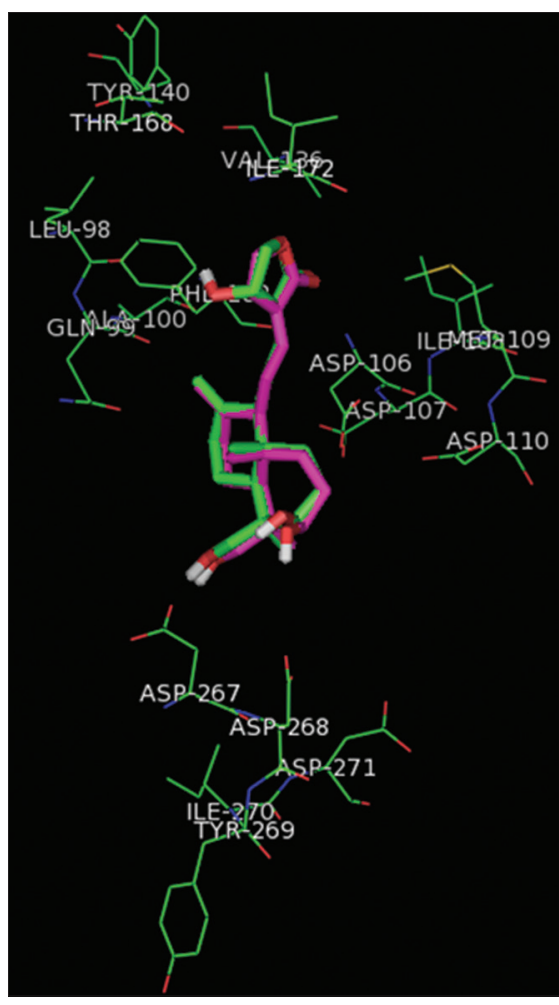

Fig. 5: Docking pose of andrographolide (green sticks) and derivative (pink sticks) in Plasmodium falciparum geranylgerany pyrophosphate synthase. The first aspartate-rich motif is on the right, and the second aspartate-rich motif is at the bottom; on the left is the hydrophobic anchor of PFGGPPS active site (image generated by PyMOL 1.3) [21] 
${ }^{13} \mathrm{C}$ NMR $\left(101 \mathrm{MHz}, \mathrm{CDCl}_{3}\right) \delta 198.53,169.88,167.27,148.10,146.89$, $146.69,142.65,142.58,124.22,124.15,123.44,108.59,108.43,108.15$, $102.34,80.19,77.46,77.34,77.14,76.82,64.15,56.72,56.00,55.70$, $55.10,55.05,52.16,42.64,42.02,38.84,38.76,37.78,37.68,36.94$, $36.88,32.77,32.73,28.04,25.63,24.38,23.68,22.81,15.22,15.18$

${ }^{1} \mathrm{H}$ NMR $\left(400 \mathrm{MHz}, \mathrm{CDCl}_{3}\right) \delta 9.68,9.59,9.56,7.50,7.24,7.14,6.97,6.89$, $6.82,6.78,6.75,6.62,6.42,6.09,6.07,6.02,5.97,5.42,5.31,5.04,4.91$, $4.77,4.76,4.71,4.54,4.45,4.38,4.33,4.30,4.12,4.09,3.66,3.45,3.40$, $3.38,3.24,3.22,2.95,2.91,2.74,2.64,2.60,2.47,2.35,2.32,2.22,2.12$, $1.91,1.89,1.73,1.66,1.49,1.45,1.41,1.35,1.32,1.26,1.22,1.16,0.81$, $0.74,0.60,0.44,-0.00$

These NMR data were consistent for the second and third experiments, suggesting a high reproducibility for the procedure. At the 5 -mmol scale, the reaction yield was $60.94 \%$.

The ${ }^{13} \mathrm{C}$-NMR peak near 200 ppm suggests that the andrographolide hydroxyl group at C-19 may have been oxidized by sodium carbonate into an aldehyde. This by-product would be anticipated, since the andrographolide hydroxyl group at C-19 is also vulnerable and selective protection had not been applied. We have to bear in mind also that protection requires more steps and may not be sufficiently green for drug synthesis [32]. The presence of this by-product should be investigated further by analytical and preparative high-performance liquid chromatography and liquid chromatography-mass spectroscopy.

\section{CONCLUSION}

A comparative modeling of PFGGPPS based on a PVGGPPS crystal structure (3PH7 chain B) was conducted, and the model was considered suitable for molecular docking. On molecular docking of andrographolide into the model, the design of an antimalarial andrographolide derivative, which is more hydrophobic than andrographolide, was proposed. Synthesis of this with a simple and green procedure was found to be reproducible at a 5-mmol scale.

\section{REFERENCES}

1. Wiser MF. Protozoa and Human Disease. United States: Garland Science; 2010

2. Elyazar IR, Hay SI, Baird JK. Malaria distribution, prevalence, drug resistance and control in Indonesia. Adv Parasitol 2011;74:41-175.

3. Jordão FM, Gabriel HB, Alves JM, Angeli CB, Bifano TD, Breda A, et al. Cloning and characterization of bifunctional enzyme farnesyl diphosphate/geranylgeranyl diphosphate synthase from Plasmodium falciparum. Malar J 2013;12:184

4. Artz JD, Wernimont AK, Dunford JE, Schapira M, Dong A, Zhao Y, et al. Molecular characterization of a novel geranylgeranyl pyrophosphate synthase from Plasmodium parasites. J Biol Chem 2011;286(5):3315-22.

5. Crowther GJ, Napuli AJ, Gilligan JH, Gagaring K, Borboa R, Francek $\mathrm{C}$, et al. Identification of inhibitors for putative malaria drug targets among novel antimalarial compounds. Mol Biochem Parasitol 2011:175(1):21-9.

6. Pholphana N, Rangkadilok N, Saehun J, Ritruechai S, Satayavivad J. Changes in the contents of four active diterpenoids at different growth stages in Andrographis paniculata (Burm.f.) Nees (Chuanxinlian). Chin Med 2013;8(1):2

7. Mishra K, Dash AP, Dey N. Andrographolide: A novel antimalarial diterpene lactone compound from Andrographis paniculata and its interaction with curcumin and artesunate. J Trop Med 2011;2011:579518.

8. Srivastava N, Akhila A. Biosynthesis of andrographolide in Andrographis paniculata. Phytochemistry 2010;71(11-12):1298-304.

9. Artz JD, Wernimont AK, Dunford JE, Schapira M, Dong AA, Zhao Y, et al. Crystal structure of Plasmodium vivax putative polyprenyl pyrophosphate synthase in complex with geranylgeranyl diphosphate. J Biol Chem 2011;286:3315-22.

10. UniProt. Available from: http://www.uniprot.org.

11. Carlton JM, Adams JH, Silva JC, Bidwell SL, Lorenzi H, Caler E, et al. Comparative genomics of the neglected human malaria parasite Plasmodium vivax. Nature 2008;455(7214):757-63.

12. Gupta A, Tripathi A, Avery M, Walker L, Tekwani B. Cloning and characterization of farnesyl pyrophosphate synthase from Plasmodium falciparum (D6, Serra Leon). Malaria J 2013;12:84

13. Larkin MA, Blackshields G, Brown NP, Chenna R, McGettigan PA, McWilliam $\mathrm{H}$, et al. Clustal $\mathrm{W}$ and clustal $\mathrm{X}$ version 2.0. Bioinformatics 2007;23:2947-8

14. Smith AB, Toder BH, Carroll PJ, Donohue J. Andrographolide: An x-ray crystallographic analysis. J Crystallogr Spectroscopic 1982;12(4):309-19.

15. ACD/Labs 12.01. Available from: http://www.acdlabs.com.

16. Kuntal BK, Aparoy P, Reddanna P. EasyModeller: A graphical interface to MODELLER. BMC Res Notes 2010;3:226.

17. RCSB Protein Data Bank. Available from: http://www.pdb.org/pdb/ home/home.do.

18. McGann MR, Almond HR, Nicholls A, Grant JA, Brown FK. Gaussian docking functions. Biopolymers 2003;68(1):76-90.

19. ImprintPocket. Available from: http://www.sts.bioengr.uic.edu/pni.

20. Chemie VA, Lanka S. Hydrogen bonding in andrographolide: 3-\{2-[Decahydro-6-hydroxy-5- (hydroxymethyl). Acta Cryst 1987:C43:530-2

21. Schrodinger LLC. The PyMOL Molecular Graphics System, Version 1.3r1. Available from: http://www.pymol.org.

22. Fletterick R, Matela R. Color-coded alpha-carbon models of proteins. Biopolymers 1982;21:999-1003

23. Jakalian A, Jack DB, Bayly CI. Fast, efficient generation of high-quality atomic charges. AM1-BCC model: II. Parameterization and validation. J Comput Chem 2002;23:1623-41.

24. Sayle R, Delany J. Canonicalization and Enumeration of Tautomers. Cambridge, United Kingdom: Innovations Computer Application; 1999.

25. Hawkins PC, Skillman AG, Warren GL, Ellingson BA, Stahl MT. Conformer generation with OMEGA: Algorithm and validation using high quality structures from the protein databank and cambridge structural database. J Chem Inf Model 2010;50:572-84

26. Halgren T. Merck molecular force field. I. Basis, form, scope, parameterization, and performance of MMFF94. J Comput Chem 1996;17(5-6):490-519.

27. Ratnani R, Hartati I, Kurniasari L. Potential production of andrographolide from sambiloto (Andrographis paniculata Nees) through the extraction of hydrotropy. Momentum 2012;8(1):6-10.

28. Suharmiati HL. Isolasi dan identifikasi Andrographolida dari herba Andrographis paniculata ness. Media Litbang Kesehatan 2001;11:33-8.

29. Lomlim L, Jirayupong N, Plubrukarn A. Heat-accelerated degradation of solid-state andrographolide. Chem Pharm Bull (Tokyo) 2003;51(1):24-6.

30. Pandeti S, Sonkar R, Shukla A, Bhatia G, Tadigoppula N. Synthesis of new andrographolide derivatives and evaluation of their antidyslipidemic, LDL-oxidation and antioxidant activity. Eur J Med Chem 2013;69:439-48.

31. Kerns EH, Di L. Drug-Like Properties: Concepts, Structure, Design and Methods. Netherlands: Elsevier; 2008.

32. Patrick G. Instant Notes in Medicinal Chemistry. United Kingdom: BIOS Scientific Publishers; 2001. 